\title{
Estudio de la influencia de la automatización en el proyecto arquitectónico
}

\section{Study of the influence of home automation in the architectural project}

\author{
$\underline{\text { S. Millán-Anglés }}{ }^{(*)}$, A. García-Santos ${ }^{(*)}$, F.J. Jiménez-Leube ${ }^{(*)}$, O. Higuera-Rincón ${ }^{(* *)}$
}

\section{RESUMEN}

La domótica integrada puede terminar jugando un papel fundamental en la arquitectura del futuro. Sin embargo, hasta hoy la automatización doméstica no es un factor tenido en cuenta desde el proyecto arquitectónico y este hecho hace que esta disciplina no se generalice para replantear el futuro de estas nuevas construcciones. Por ello, se plantea la necesidad de un estudio profundo de la influencia sobre los aspectos constructivos y proyectuales que existen en la domotización y automatización de viviendas. Para desarrollar esta investigación se han aislado los posibles condicionantes de la automatización, condicionantes que deberían tenerse en cuenta desde la fase proyecto para fomentar la implantación de las nuevas tecnologías en los edificios y así garantizar la viabilidad de estas nuevas instalaciones. El presente artículo analiza, por tanto, las principales consideraciones de carácter constructivo y proyectual que se están detectando en el estudio de la influencia de la domótica sobre la forma de concebir un proyecto arquitectónico.

Palabras clave: Automatización; domótica; Infraestructura Común de Telecomunicaciones; ICT; edificios inteligentes.

\section{ABSTRACT}

Integrated automation may have a fundamental role in the architecture of the future. However, until today the home automation is not a factor taken into account from the architectural design and this impedes this discipline to be generalised in order to reshape the future of these new buildings. Therefore, there is a need for a thorough study of the influence on constructive and projective aspects that exist in domotics and home automation. To develop this research there have been isolated the constructive and projective conditions detected in home automation, conditions that should be taken into account from the design stage to promote the implementation of new technologies in buildings in order to ensure the viability of these new facilities. This article analyzes, therefore, the main constructive and projective considerations being detected in the study of the influence of home automation on how to design an architectural project.

Keywords: Automation; domotics; Common Telecommunication Infrastructure; ICT; smart buildings.

(*) Universidad Politécnica de Madrid (España)

(*) Universidad Rey Juan Carlos, Madrid (España)

Persona de contacto/Corresponding Author: susana.millan@gmail.com (S. Millán-Anglés)

Cómo citar este artículo/Citation: Millán-Anglés, S., García-Santos, A., Jiménez-Leube, F.J., Higuera-Rincón, O. (2014). Estudio de la influencia de la automatización en el proyecto arquitectónico. Informes de la Construcción, 66(534): eo20, doi: http://dx.doi.org/10.3989/ic.12.081.

Licencia/License: Salvo indicación contraria, todos los contenidos de la edición electrónica de Informes de la Construcción se distribuyen bajo una licencia de uso y distribución Creative Commons Reconocimiento no Comercial 3.o. España (cc-by-nc). 


\section{OBJETO}

Cuando se usa el término domótica, se habla de automatizar tareas eminentemente domésticas o bien automatizar la gestión, control y supervisión de las instalaciones de un edificio de viviendas. En este contexto, sabemos que no existe domótica en la primera mitad del siglo XX, hasta entonces sólo podemos hablar de automatismos aislados pensados más o menos para su uso doméstico y cuyo grado de integración en los edificios ni siquiera se planteaba. De esta manera partimos de los primeros artefactos que no tenían una utilidad práctica o los primeros autómatas de la antigua Etiopia (1). En los diez libros de la arquitectura de Vitruvio podemos encontrar descripciones de las máquinas que se usaban en la construcción y es también muy famoso el intento del motor continuo de Villard de Honecourt (2). Pero es a partir del siglo XV, con las ilustraciones de Leonardo Da Vinci, cuando encontramos evidencias de los primeros artefactos que claramente pretenden desarrollar tareas eminentemente humanas y domésticas. Se puede decir, por tanto, que la domótica es un proceso que deriva de la secuencia (mecánica-automática-domótica). También se puede afirmar que surge cuando el ser humano se deshace de los artefactos aislados para automatizar directamente el medio en el que vive, porque, a través de la inclusión e integración de redes de información, informática, electrónica y mecanismos en la arquitectura se consigue automatizar directamente el medio en lugar de requerir elementos independientes a la misma que satisfagan estos servicios.

Los autores más conocidos (3) y que han documentado esta disciplina sitúan su origen en torno a los años 70 con los primeros dispositivos de automatización con tecnología X-10, esta tecnología define un protocolo de comunicaciones entre dispositivos eléctricos que utiliza la instalación eléctrica de baja tensión ya existente en la vivienda para conectar dichos dispositivos (4). Después, en EEUU surgieron los primeros electrodomésticos pensados específicamente para el hogar y se empezaron a instalar los primeros dispositivos que regulaban la temperatura de confort en edificios de oficinas. A finales de los 80 y con el auge mundial de los PCs aparecen los primeros sistemas de cableado estructurado que conectaban periféricos y terminales con cable estándar, fue entonces cuando empezó a usarse la expresión «edificios inteligentes» (5). La Enciclopedia Larousse en 1988 definió el término domótica, sin embargo, este hecho no contribuyó a establecer un consenso sobre el significado claro del término, sobre todo entre los diccionarios franceses, siempre precursores de los nuevos términos relacionados con la ingeniería (6). Tampoco se puede encontrar hoy día un acuerdo claro sobre la etimología de la palabra, que a menudo se explica como (domus = casa $)+$ informatique $=$ domótica, o bien como $($ domus $=$ casa $)+$ robotique $=$ domótica, o como $($ domus $=$ casa, latín $)$ + tica (automática, griego) = domótica. Como resultado, hoy día coexisten términos como «edificio domotizado» «edificio inteligente» «hogar conectado» «hogar digital»... etc. La principal razón de la proliferación de términos y nomenclaturas responde, sobretodo, a iniciativas comerciales o programas de asociaciones de estandarización que promueven la difusión de un aspecto singular de la domótica.

Por esta razón, aunque contemplaremos la existencia de dicha nomenclatura en el presente trabajo, se estimó oportuno fijar, como punto de partida, una definición que describiera satisfactoriamente el concepto de domótica y que acotara el campo de aplicación durante la investigación:
Domótica: Conjunto escalable de servicios que en grado mayor o menor se integran en la vivienda y son suministrados por sistemas que pueden configurarse en una o varias redes internas del hábitat y que, a su vez, pueden comunicarse con redes exteriores a la vivienda. Estos servicios realizan funciones relacionados con el ahorro de energía, la gestión técnica de las instalaciones, la información, la comunicación, el ocio, la accesibilidad, la asistencia, el confort... etc. Y su control puede realizarse en uno o varios puntos o centros de gestión.

De acuerdo con esta definición, se entenderá por «servicio» al conjunto de acciones automatizables que satisfacen una determinada necesidad del usuario del edificio, y por «sistema» al conjunto de componentes e infraestructura necesaria para proporcionar dichos servicios.

De la misma manera se han confeccionado y definido una serie de términos indispensables para poder guiar la investigación entre los que destacamos:

Instalación domótica integrada: Una instalación domótica integrada en la arquitectura es aquella cuyos componentes principales cumplen una doble función, la propia función del servicio que se ofrece y la arquitectónica, es decir, sustituyen o forman parte de los elementos constructivos convencionales o son elementos constituyentes de la composición arquitectónica (7).

Condicionante proyectual o constructivo: Consideración de partida que hasta a ahora no se ha tenido en cuenta en la fase de proyecto y es necesaria para poder proyectar una instalación domótica satisfactoriamente.

Existen muchas referencias que demuestran que el buen diseño de las instalaciones implica la consideración previa de decisiones sobre soluciones constructivas en proyecto. De la misma manera, muchos autores explican el por qué de otorgar la misma importancia en este sentido a las instalaciones que a la estructura del edificio (8). Una instalación domótica no se diferencia en este sentido del resto de las instalaciones del edificio, es decir, el buen diseño de esta instalación implicará la toma de consideraciones previas en fase de proyecto que afectará a los sistemas constructivos. Usando la terminología que se acaba de proponer, tener en cuenta los condicionantes constructivos de una instalación domótica, garantiza la inclusión misma de la domótica en el proyecto y evita, por ejemplo, costes derivados de modificaciones posteriores en el proyecto. Sin embargo, una diferencia fundamental de la domótica con respecto al resto de las instalaciones de un edificio es el servicio que suministra al usuario de un edificio. Mientras que en el resto de las instalaciones el servicio que se suministra es conocido y no varía significativamente de un edificio a otro, en domótica el servicio suministrado no es único sino múltiple, y puede variar sustancialmente de un edificio a otro.

Por otro lado, si nos centramos en la integración arquitectónica, además de las ventajas que ésta implica para instalaciones convencionales, en instalaciones domóticas esta integración muchas veces implica, además, la viabilidad de la propia instalación. Por ejemplo, un hueco acristalado y practicable en fachada que no se haya pensado como un «hueco móvil automatizable» con la configuración constructiva que esto implica, imposibilita la propia inclusión de un servicio domótico que se denomine «acristalamiento móvil automa- 
tizable para optimizar consumo energético o/y la renovación del aire». Por esta razón, la integración arquitectónica de una instalación domótica implica, en esencia, preestablecer los servicios automatizables que potencialmente se pueden implementar en dicha instalación.

Lo que diferencia un edificio domotizado de otro no domotizado, por tanto, será la capacidad del primero de automatizar y gestionar los procesos y servicios que en el segundo no son automatizables. El objetivo del presente trabajo no es estudiar las bondades o ventajas de domotizar edificios (9) (10), sino investigar si existen consideraciones proyectuales o constructivas que se deben prever en el proyecto arquitectónico para incluir estas instalaciones de automatización y gestión en los edificios. El interés de este estudio se centra en determinar la importancia de considerar estas instalaciones en la fase de proyecto, ya que en el caso de edificios de nueva planta dichas consideraciones son posibles, mientras que en un edificio existente, el planteamiento es muy diferente (11).

Para establecer y comprobar en cada momento las necesidades y puntos de partida de la investigación se está desarrollando, de forma paralela para esta investigación, un estudio detallado y pormenorizado de 50 empresas que desarrollan su actividad en el campo de la domótica. El objetivo de este estudio es obtener de forma preliminar resultados reales de los tipos de servicios y productos que ofrecen estas empresas; así como determinar el nivel de penetración y uso de los distintos tipos de tecnologías. Para recabar estos datos se han confeccionado unas fichas de chequeo que estandarizan los principales datos recogidos: origen y antigüedad de la empresa, tipo de empresa, tecnologías que utiliza, servicios que facilita y aspectos de integración de los servicios domóticos.

Todos los resultados de este estudio se han tabulado y graficado para establecer las comparaciones cuantitativas y cualitativas (ejemplo en la Figura 1) para poder llegar a, entre otras, las siguientes conclusiones parciales:

- La mayor parte de las empresas son instaladoras e integradoras. Sólo seis tienen más de 30 años de antigüedad y el resto tienen una vida aproximada de 10 años.
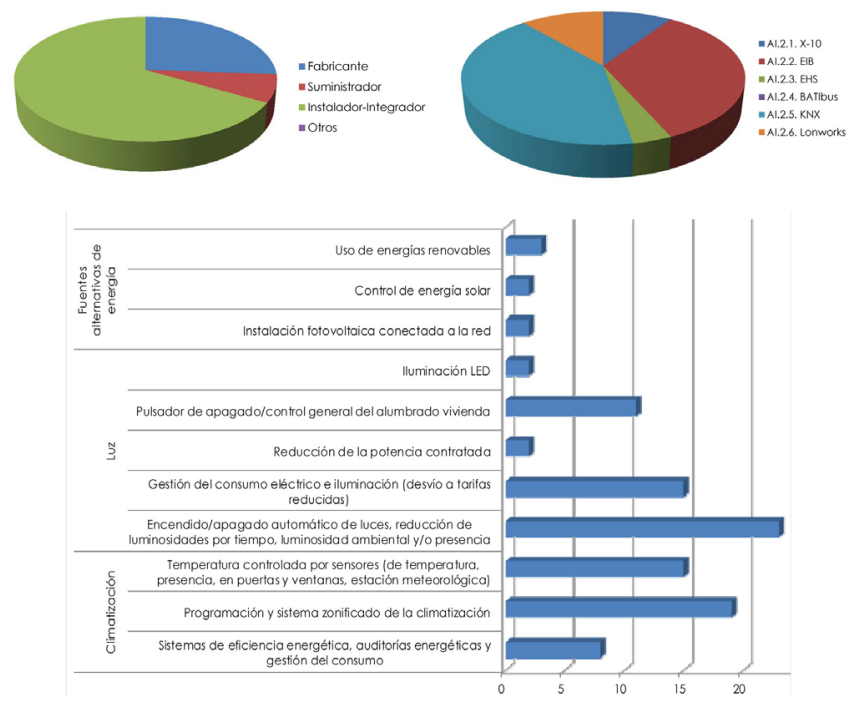

Figura 1. «Tipos de empresas encuestadas» «Protocolos de control que utilizan» $\mathrm{y}$ «número de empresas que ofertan determinados servicios pormenorizados en el campo de la eficiencia energética».
- Los protocolos de comunicación más usados son los WIFI, Ethernet y Bluetooth. En cuanto a los protocolos de control, éstos son el EIB y KNX. Las plataformas frameworks más usadas son Lonworks, Upnp y OSGI.

- Casi la totalidad de las empresas ofrecen, por orden de importancia, servicios relativos al confort, la seguridad y accesibilidad. Los otros dos grupos de servicios, eficiencia energética y comunicaciones, también son ofrecidos por la mayoría de empresas pero son todavía secundarios. Se prevé, sin embargo, que las últimas modificaciones en normativa de edificación y eficiencia energética tengan una influencia significativa en la difusión y demanda de estos servicios.

- Se puede determinar que casi todas las empresas ofrecen algún mecanismo de detección de fugas de gas, agua, humos, fallos de caldera, aumentos de temperatura y de suministro eléctrico con el correspondiente aviso (ya sea local o remoto).

- En cuanto a los servicios relacionados con las comunicaciones, todavía no llega a la mitad el número de empresas analizadas que ofrecen conexión a Internet, siendo mayor las que ofrecen, al menos, conexión remota al sistema domótico de la vivienda. Entenderemos por conexión remota la capacidad de interacción del usuario con la instalación de manera externa a la misma, por ejemplo mediante dispositivos de telefonía móvil.

- El servicio de mantenimiento y/o soporte técnico no está extendido (12), y sólo un tercio de las empresas analizadas lo ofrecen.

Sin embargo, la conclusión más significativa a la que se ha podido llegar en este aspecto es que la preinstalación es un concepto conocido y aceptado en la vivienda para servicios como el aire acondicionado o tomas de electrodomésticos pero se desconocen las características que debería tener una preinstalación para la domotización de una vivienda (13). Actualmente tan sólo prototipos de viviendas muy específicos y experimentales permiten realizar ensayos reales sobre cómo deberían proyectarse este tipo de instalaciones (14).

\section{METODOLOGÍA}

Actualmente se están desarrollando mejoras, innovaciones y avances en la construcción que se centran en muy diversas facetas del proceso constructivo, sin embargo, no todos los avances implican la introducción en la arquitectura de tecnologías que hasta hoy no eran propias de ésta. La domótica es hoy día una disciplina que, aunque tiene unos 30 años de antigüedad, aún no se ha definido a sí misma. La domótica es, además, una disciplina cuyo avance implica la introducción en la arquitectura de tecnologías completamente ajenas a ella hasta la fecha. Esta investigación parte de la hipótesis de la existencia de condicionantes constructivos que derivan de las instalaciones domóticas convencionales y que, actualmente, pueden no estar teniéndose en cuenta durante el proyecto arquitectónico. Como proyecto arquitectónico entenderemos el proceso integro de definición previo a la ejecución material de un edificio, englobando en dicho proceso, tanto las consideraciones compositivas o programáticas como las constructivas. El estudio, búsqueda y definición de estos condicionantes proyectuales o constructivos se ha realizado de la manera que sigue:

En primer lugar, y como se ha mostrado en el apartado 1. Objeto, se han estudiado los antecedentes históricos en arquitec- 
tura que dieron pie al concepto que hoy tenemos de la domótica. Después se han estudiado los conceptos relacionados con la materia, intentando dar y proponer una definición de la domótica satisfactoria para el desarrollo del trabajo y desechando conceptos inexactos o incongruentes. En la segunda parte del trabajo se presenta la necesidad de estudiar las tecnologías en las que se apoya la domótica, estas tecnologías derivan de las llamadas TIC (Tecnologías de la información). Una vez estudiadas y clasificadas estas tecnologías se ha estudiado si de ellas se derivan condicionantes constructivos. A continuación se ha estudiado la normativa que actualmente le es de aplicación a la domótica, se ha establecido si el contenido de esta normativa establece, define o reconoce condicionantes constructivos en las soluciones domóticas y se ha propuesto una revisión de esta normativa. Al final de cada apartado las conclusiones preliminares se adelantan describiendo los:

- Condicionantes constructivos detectados en la aplicación de las nuevas TIC.

- Condicionantes en edificios domotizados con conexión a redes exteriores.

- Condicionantes derivados de la normativa de aplicación.

Lo que lleva finalmente a establecer las conclusiones principales y las conclusiones derivadas del estudio. Cabe destacar que, los condicionantes detectados en estos tres estadios de la investigación no englobarían la totalidad de los condicionantes que cabe tener en cuenta en un edificio domotizado, existen otros condicionantes que derivan de otros servicios no contemplados en estos tres estadios y para los cuales habrá que determinar en fase de proyecto qué condicionantes constructivos implican. Por ejemplo, un edificio en el que se plantee una tabiquería móvil, los condicionantes constructivos para este servicio tendrán que ver, por ejemplo, con el tipo de tabiquería que puede hacer este servicio posible.

La investigación, por tanto, se ha enfocado desde el punto de vista del arquitecto o proyectista, es decir desde el punto de vista de la concepción y diseño del proyecto, en aras de que dicho proyectista se convierta en conocedor consciente de estas nuevas tecnologías y las emplee a la hora de pensar los nuevos edificios.

\section{DISTINCIÓN ENTRE LOS CONDICIONANTES DERIVADOS DE LAS NUEVAS TECNOLOGÍAS Y CONDICIONANTES DERIVADOS DE LOS COMPONENTES DE LAS INSTALACIONES DOMÓTICAS}

En un edificio domotizado tenemos insertada una red de datos de la misma naturaleza o muy similar a las redes de computadoras. Por esta razón, estamos estudiando tecnologías y disciplinas poco habituales para la figura del arquitecto pero necesarias para entender y aprehender la domótica. En la investigación y búsqueda los condicionantes constructivos se ha incluido, por tanto, el estudio de las principales tecnologías implicadas o trascendentes hoy día en el campo de la domótica (15). Estas tecnologías, que derivan de la ingeniería informática y de telecomunicación, se han dividido en los siguientes tipos:

- Tecnologías que definen protocolos de comunicaciones.

- Tecnologías que definen protocolos de control.

- Tecnologías que definen plataformas o Frameworks y middelwares.
Lo que hace agrupar estas tecnologías en estos tres grupos son el nivel de abstracción o el nivel al que trabajan en la pila de capas de la arquitectura de la red. De esto se extrae que la división de estos tres grupos o tipos de tecnologías no es exacto ni inflexible pero es una herramienta útil para encuadrar el campo de actuación de las tecnologías que se usan en domótica.

Si nos centramos en los condicionantes constructivos de las diferentes tecnologías domóticas, y dejando de lado los condicionantes específicos de los dispositivos, se puede decir que:

- Es en la instalación de la red de interconexión donde encontramos mas condicionantes, además de en la ubicación de los dispositivos de control, que dependerá de si incorporan la interfaz hombre-máquina (16).

- En función de la tecnología escogida será necesario o no contemplar un cableado específico de interconexión o bien podremos aprovechar un cableado existente de otra red del inmueble (17). En cualquiera de estos casos, el condicionante que varía es la reserva de espacio, los recorridos y topología del cableado. Cabe destacar que en este sentido, el condicionante de reserva de espacio aunque presente, es menos importante que el condicionante de recorridos, es decir, lo realmente relevante es que los elementos constructivos permitan el trazado mismo de la topología de cableado propuesta.

- Los nuevos aparatos y dispositivos que aparecen ahora en las viviendas ponen en tela de juicio la configuración y el diseño de las actuales instalaciones eléctricas. Como se comentará más adelante con más detalle, comienza a detectarse la necesidad del replanteamiento de esta instalación para estudiar, por ejemplo, los posibles beneficios de incluir un circuito de electricidad en corriente continua y voltaje moderado que alimente a estos nuevos dispositivos.

- En cuanto a los dispositivos (sensores, actuadores, transformadores...etc.) El condicionante inmediato es la reserva de espacio que estos requieren y saber si estos se sitúan empotrados o superpuestos en los paramentos y superficies. Aunque existen elementos empotrables, la integración de estos elementos no va actualmente más allá.

Los nodos de una instalación domótica que gestionan los servicios automatizables hacen posible que estos servicios se autoajusten, de forma inteligente, a medida que el sistema se retroalimenta con los datos sobre las relaciones entre acciones y resultados. Sin embargo, hoy día este hecho tiene como consecuencia tan solo la depuración del servicio en particular para el edificio en concreto y en uso. El mantenimiento y seguimiento de estas instalaciones por empresas especializadas permitiría el uso de esta retroalimentación para proponer nuevas consideraciones para instalaciones futuras, hecho importante ya que estas consideraciones podrían tener influencia en los condicionantes constructivos. Sin embargo, este seguimiento no está extendido hoy día y dicha retroalimentación no se está considerando de manera significativa en el diseño de instalaciones domóticas en edificios de nueva planta.

En cuanto a la interfaz hombre-máquina, que define la manera en la que el usuario accede gestión de los servicios domóticos, existen hoy día múltiples estudios que describen, sobre todo, la accesibilidad de dicha interfaz, la amigabilidad de la misma o su manejo intuitivo (5) (18). Sin embargo, no parece 
haber consenso sobre la ubicación de la misma. Esta interfaz se propone a menudo a través de la pantalla de televisor de la vivienda, en el hall de entrada, o preferentemente a través de dispositivos móviles. Tampoco hay consenso sobre el hecho de si esta interfaz debe ser única para todos los servicios de la vivienda o deben ofrecerse interfaces diferenciadas en función del tipo de servicio, si bien es cierto que la existencia de tecnologías bajo patente o «tecnologías propietario» para determinados servicios obliga a menudo a interfaces independientes del resto de servicios debido a incompatibilidades derivadas de dicha patente (19). En cualquier caso, la definición de la interfaz y su ubicación puede afectar a la configuración programática de la arquitectura ya que cualifica espacios de dentro de la misma.

\section{CONDICIONANTES ESPECÍFICOS DE EDIFICIOS DOMOTIZADOS CON CONEXIÓN A REDES EXTERIORES}

Un edificio con instalación domótica es un edificio provisto de una o varias redes internas que automatizan una serie de servicios. Estas redes pueden o no estar conectadas a su vez a redes de telecomunicaciones externas al edificio. Por esta razón ha sido necesario en esta investigación buscar también condicionantes constructivos o proyectuales derivados de esta posible conexión con redes exteriores.

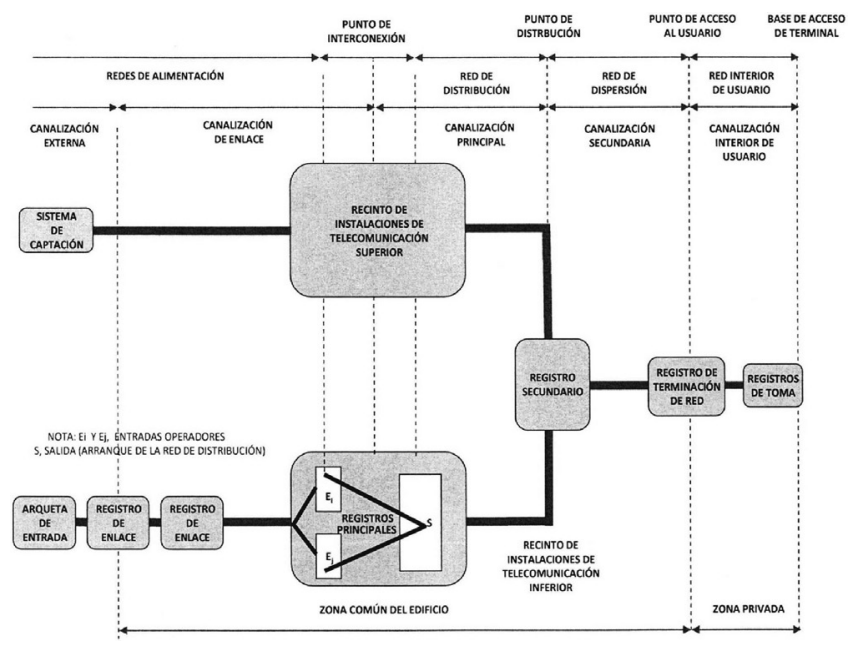

Figura 2. «Esquema general de una I.C.T.» (Reglamento I.C.T.).

Una vivienda domótica no tiene que estar conectada a redes exteriores para considerarla como tal, pero sí juega esta conexión a redes exteriores un papel importante en las posibilidades de los nuevos servicios domóticos. En cuanto a los condicionantes constructivos y proyectuales detectados y analizados en este caso, las principales conclusiones han sido:

- La infraestructura de la ICT (Infraestructura Común de Telecomunicación) (20) lleva aparejados fuertes condicionantes que afectan sobre todo a las zonas comunes de un edificio de viviendas o a conjuntos de edificios de viviendas. Es necesario en este caso prever grandes reservas de espacios y ubicaciones de cuartos específicos que requieren unas condiciones técnicas determinadas (Figura 2).

- Podemos distinguir claramente entre las redes comunes del edificio y la red del usuario que empieza a partir del PAU (Punto de acceso a usuario). En el interior de la vivienda, a su vez, cabe prever tanto el recorrido de la dotación mínima de tomas de acceso como la previsión y reservas de canalizaciones.

- Se contempla una interacción de redes de cableado diferenciada que realmente no lleva aparejada confluencia o incompatibilidad de oficios o responsabilidades. Es decir, aunque existen secciones de cableado cuyo mantenimiento o responsabilidad responde a diferentes agentes y/o empresas (Figura 3), estas secciones se encuentran correctamente diferenciadas y la interconexión entre ellas se identifica mediante un tipo de nodo con unas características específicas. Tampoco parece haber incompatibilidades a la hora de usar canalizaciones de manera conjunta con la red de suministro eléctrico del edificio. En el RITI o RITS se define el punto hasta el cual la empresa suministradora es responsable de la instalación y el PAU define el punto en el que comienza la red interior de la vivienda particular.

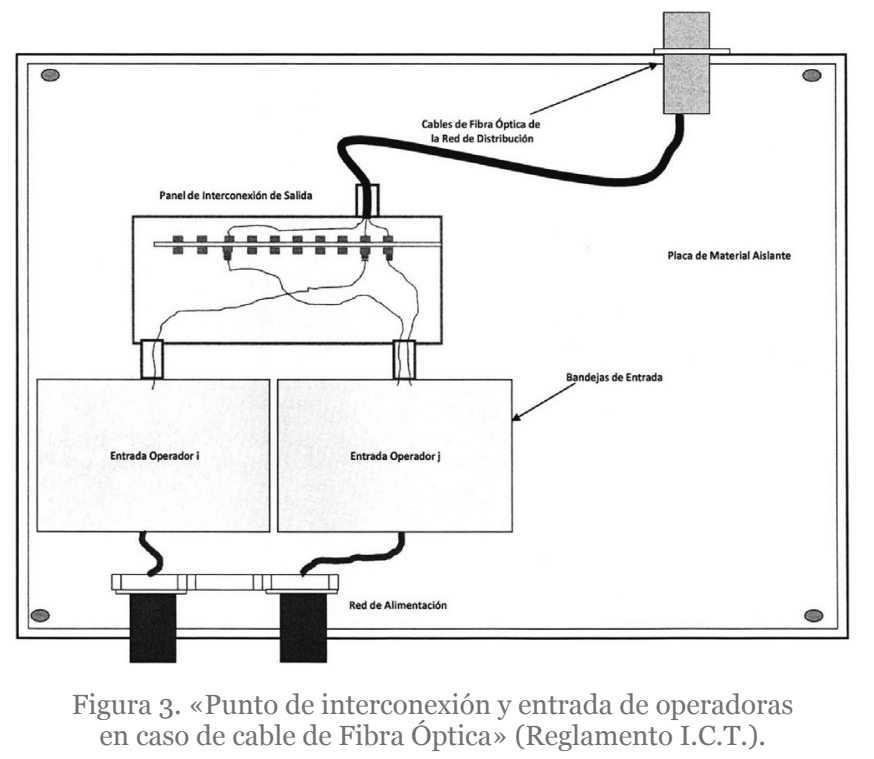

\section{CONDICIONANTES DERIVADOS DE LA NORMATIVA DE APLICACIÓN}

La normativa de aplicación es la encargada de establecer, para cada aspecto del proceso edificatorio, las exigencias técnicas que se han de satisfacer en el proyecto. Lo que se suele desconocer y a menudo obviar, es la capacidad que tiene la normativa para fomentar e impulsar el uso de determinadas soluciones constructivas. El estudio CEDOM realizado en el 2011(21) detalla como la normativa ha jugado en España un papel especial en el impulso del grado de penetración de las soluciones de automatización en edificios a pesar de la desaceleración de la actividad edificatoria en los últimos años.

No existe actualmente una normativa que regule específicamente las instalaciones domóticas, sin embargo, si existen dos reglamentos estatales o nacionales que tratan cada uno parcialmente el tema de los automatismos para viviendas. Por un lado tenemos la I.C.T. (Normativa de las infraestructuras Comunes de Telecomunicación) que regula las infraestructuras comunes de telecomunicación para el acceso a los servicios de telecomunicación en el interior de los edificios y de la actividad de instalación de equipos y sistemas de telecomunicaciones. Sin embargo, el reglamento I.C.T. no regula las infraestructuras (canalizaciones y cableado) necesarias en el hogar para la instalación de los sistemas domóticos, entendiendo como tales los sistemas de automatización, gestión de 
la energía confort y seguridad en viviendas y edificios. Estos Sistemas tienen su regulación en la R.E.B.T. (Reglamento Electrotécnico de Baja Tensión) (22) este reglamento debe establecer las condiciones técnicas y las garantías necesarias que deben reunir los sistemas que realizan una función de automatización. No obstante, en cuanto a alimentación eléctrica de los equipos (protecciones, líneas, tomas, etc.) la R.E.B.T. no especifica apenas nada, salvo remitir a la ICT-36 «Instalaciones de Muy Baja Tensión». Para encontrar «requisitos específicos de la instalación de los sistemas de automatización para viviendas y edificios» (23) hay que acudir a un documento que, aunque no es vinculante y por tanto no es de obligado cumplimiento, es una extensión de la REBT. Se trata de la Guía Técnica de aplicación de la REBT, concretamente el apartado GUÍA-BT-51. Cabe destacar, además, que esta guía propone realizar una preinstalación domótica en base a unas recomendaciones para la instalación de estos sistemas.

Con respecto al cableado de datos, éste se empezó a regular con la normativa ICT aunque por ahora no se ha planteado la posibilidad de instalar o prever una línea eléctrica específica que alimente a estos los dispositivos. Si hace unos años la mayoría de los electrodomésticos y sistemas de iluminación en una vivienda se alimentaban directamente de la instalación eléctrica de la vivienda, en la actualidad un porcentaje amplio del consumo eléctrico son dispositivos conectados a través de adaptadores de 5 ó 12 VDC (ordenadores, teléfonos móviles, sistemas de iluminación basados en LEDS,...) Por tanto, parece conveniente estudiar la conveniencia de disponer en viviendas de líneas de alimentación continua. Las viviendas actuales se alimentan con energía eléctrica a 220 VAC en Europa y a 120 VAC en Norteamérica, sin embargo, muchos dispositivos y entre ellos la pequeña aparamenta asociada a la domótica requieren la energía entre los 5 y los 24 VDC.

Si en las viviendas se preinstalara una línea eléctrica específica se podrían alimentar los dispositivos domóticos y se podría prescindir de la existencia de baterías y del mantenimiento de las mismas. Hoy día sólo la instrucción ITC-BT-25 de la REBT contempla la necesidad de la inclusión de un circuito adicional para sistemas de automatización y solo en caso de viviendas de electrificación elevada, sin especificar condición o exigencia diferenciada alguna para este circuito.

Existen otras iniciativas de revisión de la normativa como la ICT-T de Telefónica, sin embargo, estas iniciativas son hoy día reflexiones con un marcado carácter comercial y no constituyen documentos de carácter vinculante, por lo que suelen representar un enfoque que quizá no se ha revisado lo suficiente. Si bien es cierto, cabe señalar, que este tipo de iniciativas promueven la reflexión sobre lo que se puede o no regular desde las normativas que si tienen carácter vinculante.

Si la normativa I.C.T. regula las Infraestructuras Comunes de Telecomunicaciones y la R.E.B.T. caracteriza actualmente los sistemas de automatización habría que delimitar claramente este hecho. Aunque desde el 2005 la normativa de aplicación a estas instalaciones ha comenzado a establecerse, la evolución de esta normativa propiciaría aún más implantar este tipo de instalaciones en las viviendas por lo que siempre parece preceptivo proponer una revisión de la actual R.E.B.T. ampliada. En este sentido se propone:

- Esta revisión normativa debería incluir garantías de estandarización de dispositivos y tecnologías que garantizase la compatibilidad, difusión y asequibilidad de estas nuevas soluciones domóticas.

- Dicha revisión debería abordar el problema de la actualización o rehabilitación de instalaciones en edificios existentes

- Parece conveniente que la nueva R.E.B.T. ampliada tratara la automatización en las viviendas no sólo desde el punto de vista energético y de gestión técnica o seguridad sino en un concepto mucho más amplio de forma que no se excluyan otros servicios relacionados con el ocio o el confort. Para conseguir tal fin, este reglamento debería incluir, en su cuerpo normativo, al menos los requisitos específicos contenidos en el apartado GUÍA-BT-51 de su Guía Técnica de Aplicación.

- Se hace patente la necesidad de replantear la estructura actual de la red eléctrica doméstica.

- Al margen de lo especificado en la GUÍA-BT-51, hay que hacer notar que no existen hoy día otras recomendaciones o sugerencias en estas normativas o sus revisiones comerciales (ICT-T) orientadas a los arquitectos y que determinen o reconozcan la interacción de las redes con la arquitectura.

\section{CONCLUSIONES}

Tras la investigación expuesta se han obtenido las siguientes conclusiones principales:

- Existen condicionantes constructivos y de proyecto derivados de la inclusión de las instalaciones de automatización en los proyectos de viviendas.

- El arquitecto debe tener en cuenta estos condicionantes para proyectar edificios domotizados de forma satisfactoria ya que son variables que cabe considerar en fase de proyecto. No sólo con el objetivo de evitar costes por imprevisión de los mismos, de potenciar al máximo las posibilidades de la automatización del edificio o prevenir incompatibilidades constructivas o estéticas, sino para garantizar también la propia viabilidad de la instalación domótica.

- El mantenimiento es actualmente un servicio que no ofrecen las empresas de manera generalizada y que es un factor importante ya que incide sobre la confianza del usuario a la hora de demandar una instalación domótica. Además, si este servicio se generalizase, se podrían utilizar los datos que generan muchos de los subsistemas de una instalación domótica para evaluar resultados. Esto ayudaría a ajustar futuras instalaciones e incluso revisar posibles condicionantes constructivos o proponer nuevos.

Si entendemos como "preinstalación» la consideración previa en fase de proyecto de ciertos condicionantes constructivos comunes a cualquier edificio, entonces podemos afirmar que una preinstalación domótica es posible. Esta preinstalación domótica, por tanto, no elimina los condicionantes constructivos, sino que los considera de forma previa a través de determinadas configuraciones constructivas que garantizan que esos condicionantes se tienen en cuenta, facilitando que una instalación domótica se implemente con éxito en un edificio. A partir de este punto, cabe plantear la necesidad de estudiar cuales son los condicionantes englobables en dicha «preinstalación domótica» y en qué configuraciones constructivas se traduce dicha preinstalación.

Asimismo, se extraen las siguientes conclusiones derivadas

- El tipo de tecnología escogido determina la topología de la red interna de la vivienda, si necesita o no cableado es- 
pecífico e incluso la relación entre los dispositivos, repetidores, etc.

- La previsión de espacios es el condicionante más importante en relación a la conexión de las redes internas de la vivienda con redes exteriores del edificio.

- Aunque no existen guías prácticas para el arquitecto estos condicionantes espaciales se podrían sistematizar y tabular en documentos actualizables que guiaran los proyectos de edificación.

- Es recomendable una revisión de la R.E.B.T en coordinación con la I.C.T. que complete las garantías que debe reunir las canalizaciones y el cableado aparejados a los servicios domóticos. Y que contemple el concepto de «preinstalación» de una manera vinculante.

Los principales avances propuestos en la materia son los siguientes:

- La interfaz hombre máquina está por definir desde el punto de vista de la cualificación de espacios en la vivienda.
- Es necesario replantear la instalación eléctrica de la vivienda y evaluar la procedencia de nuevos circuitos eléctricos que alimentarían los nuevos dispositivos.

Como reflexión final se ha comprobado que el cambio cualitativo que debe dar la domótica es el de su integración, entendiendo como tal la fusión entre servicio y elemento constructivo. Además, tras determinar que proyectar un edificio domotizado implica la consideración previa de ciertos condicionantes constructivos, es el proyectista el agente cualificado para establecer, en fase de proyecto, qué soluciones constructivas satisfacen o contemplan dichos condicionantes (24). Por esta razón, se considera de vital importancia que el arquitecto sea conocedor cualificado de la influencia de las nuevas tecnologías en los nuevos edificios domotizados.

\section{AGRADECIMIENTOS}

A mis tutores de doctorado Alfonso y Javier y a Óscar que nunca deja de amplificar mis metas.

\section{REFERENCIAS}

(1) Universidad de Zaragoza. (2010). Autómatas en la historia, http://automata.cps.unizar.es/Historia/Webs/automatas_ en_la_historia.htm.

(2) de Honnecourt, V. (2001). Cuaderno de dibujos. Madrid: Ediciones Akal.

(3) Martín Domínguez, H., Sáez Vacas, F. (2006). Domótica, un enfoque sociotécnico. Segovia: Fundación Rogelio Segovia para el desarrollo de las Telecomunicaciones.

(4) Boscán, N., Villalobos, R. (2009). Protocolos de control de dispositivos domóticos: Análisis de patentes. Télématique: Revista Electrónica de Estudios Telemáticos, 8(2): 1-14.

(5) Huidobro-Moya, J. M., Millán-Tejedor, R. J. (2004). Domótica, Edificios inteligentes (10 ed.). Madrid: Creaciones Copy Right.

(6) Flórez-de la Colina, M. A. (2004). Hacia una definición de la domótica. Informes de la Construcción, 56(494): 11-17, doi: http://dx.doi.org/10.3989/ic.2004.v56.i494.444.

(7) Ministerio de Fomento. (2013). Código Técnico de la Edificación y Documentos Básicos, http://www.codigotecnico.org/ web/recursos/documentos/.

(8) Martín-Gómez, C. (2006). Las instalaciones y la arquitectura. Tectónica: monografías de arquitectura, tecnología y construcción, (21): 4-27.

(9) García-S., Matachana, E., Lopez-Peñalver, F. J. (2004). El estado del arte de la tecnología al servicio de la construcción. Informes de la Construcción., 56(494): 19-26, doi: http://dx.doi.org/10.3989/ic.2004.v56.i494.445.

(10) Ferreira-Gili, M. (2004). Camino al hogar inteligente. Informes de la Construcción., 56(494): 5-10, doi: http://dx.doi. org/10.3989/ic.2004.v56.i494.443.

(11) Peccis-Rubio, N. (2011). Rehabilitación de viviendas eficientes e inteligentes: sistemas domóticos aplicados sobre las instalaciones térmicas de las viviendas construidas (Trabajo fin de Máster). Madrid: Escuela Universitaria de Arquitectura Técnica - Universidad Politécnica de Madrid.

(12) Soberanes-Collado, M. J. (2011). El mantenimiento de un edificio inteligente, http://esdaiposgrados.wordpress. com/2011/04/o6/el-mantenimiento-de-un-edificio-inteligente/.

(13) Asociación Española de la Domótica (CEDOM). (2008). Instalaciones domóticas, cuaderno de buenas prácticas para promotores y constructores. Barcelona: AENOR ediciones.

(14) Caamaño-Martín, E., Neila-González, J., Jiménez-Leube, F.J., Egido-Aguilera, M.A., Uzquiano, M.J., Gómez-Osuna, J.M., Bedoya-Frutos, C., Magdalena-Layos, L., García-Santos, A. (2004). Viviendas solares autosuficientes: participación de la Universidad Politécnica de Madrid en el concurso «Solar Decathlon». Informes de la Construcción, 56(494): 35-46, doi: http://dx.doi.org/10.3989/ic.2004.v56.i494.447.

(15) Tanenbaum, A. (2003). Redes de computadoras. Mexico: Pearson Education.

(16) Moreno-Navarro, I., Martín-Candelario, E., Álvarez-Alonso, M. (1999). Métodos de control en sistemas domóticos: últimas tendencias en sistemas distribuidos. Informes de la Construcción, 50(459): 43-53, doi: http://dx.doi.org/10.3989/ ic.1999.v50.i459.830.

(17) Bujdei, C., Moraru, S. (2010). Wireless communications standards for Intelligent Buildings. En Annals of DAAAM for 2010 \& Proceedings of the 21st International Symposium, 21(1).

(18) Álvarez, M., Arquero, Á., Martínez, E., Río, O. (2010). Domogis: prototipo de un interfaz del sistema de control de un edificio integrado en un SIG. Informes de la Construcción, 62(518): 15-24, doi: http://dx.doi.org/10.3989/ic.08.034.

(19) Wang, S., Xu, Z., Li, H., Hong, J., Shi, W.Z. (2004). Investigation on intelligent building standard communication protocols and application of IT technologies. Automation in Construction, 13(5): 607-619, doi: http://dx.doi.org/10.1016/j. autcon.2004.04.008. 
(20) Ministerio de Industria Energía y Turismo. (2011). Normativa de las infraestructuras Comunes de Telecomunicaciones, (I.C.T.), Orden ITC/1644/2011, de 10 de junio. http://www.minetur.gob.es/telecomunicaciones/infraestructuras/paginas/legislacion.aspx.

(21) CEDOM. (2012). Estudio CEDOM 2011, Tendencias del mercado español de domótica e inmótica. Asociación Española de Domótica.

(22) Ministerio de Industria Energía y Turismo. (2002). Reglamento de Baja Tensión, (R.E.B.T.) http://www.f2i2.net/legislacionseguridadindustrial/LegislacionNacionalGrupo.aspx?idregl=76.

(23) Ministerio de Industria Energía y Turismo. (2012). Guía Técnica de Aplicación al Reglamento de Baja Tensión, http:// www.f2i2.net/legislacionseguridadindustrial/rebt_guia.aspx.

(24) Vakilinezhad, R., Mofidi, S. M., Faizi., M. (2010). Intelligent Building Materials and Systems. The International Journal of Technology, Knowledge and Society, 6(5): 119-130. 